\title{
ONE-SIDED IDEALS IN NEAR-RINGS OF TRANSFORMATIONS
}

\author{
H. E. HEATHERLY
}

(Received 23 September 1969: revised 16 March 1970)

Communicated by B. Mond

\section{Introduction}

Let $(G,+)$ be an arbitrary group and let $T_{0}(G)=\{f \in \operatorname{Map}(G, G): 0 f=0\}$; the system composed of $T_{0}(G)$ and the operations of pointwise addition and composition of functions form a (left) near-ring. Berman and Silverman, in their investigation of near-rings of transformations [3], found that for every group $G$ the associated near-ring of transformations $T_{0}(G)$ has no proper ideals. In the present paper left and right ideals of $T_{0}(G)$ are considered.

D. W. Blackett [4] investigated one-sided ideals in $T_{0}(G)$ for $G$ finite. Blackett's results appear as consequences of the more general study in this paper. We show that for $G$ finite $T_{0}(G)$ is the direct sum of minimal right ideals, but this does not hold for $G$ infinite. Minimal one-sided ideals will be characterized completely. Maximal one-sided ideals are considered; every right (left) ideal is contained in a maximal right (left) ideal. A correspondence between normal subgroups of $G$ and left ideals in $T_{0}(G)$ is given. The center of $\left(T_{0}(G),+\right)$ is found to be a left ideal.

\section{Preliminaries on near-rings}

An algebraic system $(N,+, \cdot)$ is a (left) near-ring if

(1) $(N,+)$ is a group, not necessarily abelian,

(2) $(N, \cdot)$ is a semigroup,

(3) $c(a+b)=c a+c b$ for each $a, b, c \in N$.

In this paper it shall further be required that zero is a two-sided annihilator. This is not a consequence of (1)-(3).

$T_{0}(G)$ serves as a motivational example of a near-ring. Furthermore every near-ring can be embedded in a $T_{0}(G)$ for some $G$ [5].

A group $G$ together with a mapping $(g, r) \rightarrow g r$ of $G \times N$ into $G$ such that for $x \in G$ and $n, m \in N$

(1) $x(n+m)=x n+x m$

(2) $x(n m)=(x n) m$ 
is a near-ring module over the near-ring $N$. Note, it follows that if $0 \in G, a \in N$, then $0 a=0$.

Near-ring homomorphism and $N$-homomorphism are defined as usual. Ideals (submodules) are exactly the kernels of homomorphisms ( $N$-homomorphisms). It is well known that an ideal of $N$ is a subset $M$ such that

(1) $(M,+)$ is a normal subgroup of $(N,+)$

(2) $n m \in M$ for each $n \in N, m \in M$

(3) $\left(n_{1}+m\right) n_{2}-n_{1} n_{2} \in M$ for each $m \in M, n_{1}, n_{2} \in N$.

Subsets satisfying (1) and (2) are left ideals of $N$ and subsets satisfying (1) and (3) are right ideals of $N$. A subgroup $B$ of $(N,+)$ is an $N$-subgroup (left $N$-subgroup) if $b \in B$ and $n \in N$ implies $b n \in B(n b \in B)$. Every right ideal is a $T_{0}(G)$-subgroup, but the converse does not hold.

\section{Right ideals in $T_{0}(G)$}

Let $S$ be a non-empty subset of $G$ and define $A(S)=\left\{\alpha \in T_{0}(G): S \alpha=0\right\}$. These annihilating sets, $A(S)$, are right ideals of $T_{0}(G)$. For convenience let $P_{x}=A(G-\{x\}), x \neq 0 . P_{x}$ may also be described as $\{\alpha(x, y): y \in G\}$, where $t \alpha(x, y)=0$ if $t \neq x$ and $x \alpha(x, y)=y$. The following properties of the $\alpha(x, y)$ follow immediately:

Property A. If $y \neq s \neq 0$, then $\alpha(x, y) \alpha(s, t)=\alpha(x, 0)=0$ and $\alpha(x, s) \alpha(s, t)=\alpha(x, t)$.

Property B. The set $\{\alpha(x, x): x \in G-\{0\}\}$ is a collection of pairwise orthogonal idempotents.

Property C. 1) $\alpha(x, y)+\alpha(x, v)=\alpha(x, y+v)$

2) $-\alpha(x, y)=\alpha(x,-y)$

3) $\alpha(x, y)-\alpha(x, v)=\alpha(x, y-v)$.

Property D. For $\beta \in T_{0}(G), \alpha(x, y) \beta=\alpha(x, y \beta)$.

For any $\alpha(x, y) \in P_{x}$ we have

$$
\alpha(x, x) \alpha(x, y)=\alpha(x, y)
$$

and hence $P_{x}$ is generated by the idempotent element $\alpha(x, x)$. If $y \neq 0$, then

$$
\alpha(x, y) \alpha(y, x)=\alpha(x, x)
$$

and hence each $\alpha(x, y)$ generates $P_{x}$. We have established

THEOREM 1. For each $x \neq 0, P_{x}$ is a minimal right ideal and a minimal $T_{0}(G)$ subgroup. $P_{x}$ is generated by the idempotent $\alpha(x, x)$ which acts as a left identity for $P_{x}$. 
It is easy to identify the groups $\left(P_{x},+\right)$. The mapping $g \rightarrow \alpha(x, g)$ is a group isomorphism, hence $P_{x} \cong G$.

Let $x \in G-\{0\}$. Because of Theorem $1 P_{x} \cap \sum_{y} P_{y}=(0)$, where $y \in G-\{0, x\}$. Thus the sum of the $P_{x}$ is a (group) direct sum. For convenience denote this direct sum by $P$.

THEOREM 2. $P$ is a right ideal of $T_{0}(G)$. This theorem is a consequence of

LEMMA 0. If $F$ is a family of right ideals from a near-ring $N$, then $\Sigma M, M \in F$, is a right ideal of $N$.

Proof. It is well known that $\Sigma M$ is a normal subgroup of $(N,+)$. Let $m=\sum_{i=1}^{k} m_{i}$, where $m_{i} \in M_{i} \in F$, be any element in $\Sigma M$. The proof that

$$
\left(n_{1}+m\right) n_{2}-n_{1} n_{2} \in \Sigma M,
$$

for each $n_{1}, n_{2} \in N$, is by induction on $k$. The case $k=1$ being obvious we proceed to the inductive step. Let $c_{j}=\sum_{i=1}^{j} m_{i}$. Note that

$$
\left(\left(n_{1}+c_{k-1}\right)+m_{k}\right) n_{2}-\left(n_{1}+c_{k-1}\right) n_{2} \in M_{k}
$$

and that $\left(n_{1}+c_{k-1}\right) n_{2}-n_{1} n_{2} \in \Sigma M$ by the induction hypothesis. So $\left(n_{1}+m\right) n_{2}-n_{1} n_{2}$ is in $\Sigma M$.

Note that if $G$ is finite, say $|G|=n$, then $T_{0}(G)$ and $P$ have the same cardinality, $n^{n-1}$. A consequence of these remarks is the following theorem originally arrived at by Blackett $[4$, p. 39] in a different fashion.

THEOREM 3. If $G$ is finite, then $T_{0}(G)$ is the direct sum of the minimal right ideals.

This theorem is not true for $G$ infinite. This can be seen in a variety of ways, one of the simpler being to examine cardinality: $\left|T_{0}(G)\right|=|G|^{|G|}$, while $|P|=|G|$.

The following four lemmas lead a complete classification of minimal right ideals in $T_{0}(G)$. In the following $M$ is a non-zero right ideal of $T_{0}(G)$.

Lemma 1. For each $x \in G$, there exists $\beta \in M, t \in G$, such that $t \beta=x$.

LEMma 2. There exists non-zero $p \in M, t \in G$ such that $t p=0$.

Proof. Let rng $\beta=\{x \beta: x \in G\}$. We consider two cases.

CASE 1. There exists $\beta \in M$ such that $|\mathrm{rng} \beta|>2$. Then there exists non-zero $x, t \in G, x \neq t$, such that

$$
t \beta=y \neq z=x \beta \neq 0 .
$$

Choose $\gamma_{1}, \gamma_{2} \in T_{0}(G)$ so that

$$
(t) \gamma_{1}=-y, \quad(x) \gamma_{1}=-z,(-z) \gamma_{2}=-z,(-y) \gamma_{2}=0 .
$$

Let $p=\left(\gamma_{1}+\beta\right) \gamma_{2}-\gamma_{1} \gamma_{2}$. Then $x p=z \neq 0$ and $t p=0$. 
Before turning to the case where $|\operatorname{rng} \beta|=2$ we dispose of the special situation $|G|<4$. Since $M$ is non-zero, $|G|=1$ is not under consideration. For $|G|=2, T_{0}(G)$ itself is the only minimal right ideal. Finally for $|G|=3$ the minimal right ideals are:

$$
\{(0,0,0),(0,0,1),(0,0,2)\} \text { and }\{(0,0,0),(0,1,0),(0,2,0)\} \text {, }
$$

where $\left(x_{0}, x_{1}, x_{2}\right)$ represents the function on $C_{3}$ that takes $i$ into $x_{i}$. In each of these two cases the lemma is easily seen to be satisfied. For the remainder of the proof take $|G| \geqq 4$.

CASE 2. For each non-zero $\beta \in M,|\operatorname{rng} \beta|=2$. Let $\beta$ be a non-zero element of $M$. Then there is a non-zero element $x$ of $G$ such that $(x) \beta=y \neq 0$. Let $t$ be a second non-zero element of $G$. Choose $\gamma_{1}, \gamma_{2} \in T_{0}(G)$ such that

$$
\begin{aligned}
& (x) \gamma_{1}=-y,(t) \gamma_{1}=z \text { where } z \text { is not } 0,-y, \text { nor }-y-y, \\
& (-y) \gamma_{2}=-y, z \gamma_{2}=0, \text { and }(z+y) \gamma_{2}=(z+t \beta) \gamma_{2}=0 .
\end{aligned}
$$

Then $\left(\gamma_{1}+\beta\right) \gamma_{2}-\gamma_{1} \gamma_{2}$ is the desired mapping $p$.

Lemma 3. If $M$ is a minimal right ideal, then $G=G_{1} \cup G_{2}$, where

$$
G_{1} \cap G_{2}=\emptyset, \quad 0 \in G_{1}, G_{2} \neq \emptyset, G_{1} M=\{0\},
$$

and for each $x \in G_{2}, x \beta \neq 0$ for each non-zero $\beta \in M$.

Proof. There exists non-zero $x \in G, \beta \in M$ such that $x \beta=0$. The set $\{\gamma \in M: x \gamma=0\}$ is a non-zero right ideal of $T_{0}(G)$ and hence is equal to $M$. Let

$$
G_{1}=\{t \in G: t M=\{0\}\} \text { and } G_{2}=G-G_{1} .
$$

It is easily seen that $G_{1}$ and $G_{2}$ are the required sets.

Lemma 4. If $M$ is a minimal right ideal, then $|\mathrm{rng} \beta|=2$ for every non-zero $\beta \in M$. Hence $G_{2}$ is a singleton.

Proof. Suppose $\beta \in M$ and $0, y_{1}$, and $y_{2}$ are distinct elements of the range of $\beta$. Choose $\gamma \in T_{0}(G)$ so that $t \gamma=0$ if $t \neq y_{2}, y_{2} \gamma=y_{2}$. For some $x_{1} \in G, x_{1} \beta=y_{1}$, hence $x_{1} \beta \gamma=0$. Since there exists $x_{2} \in G$ such that $x_{2} \beta=y_{2}$ and therefore $x_{2} \beta \gamma=y_{2}$ we have that $\beta \gamma$ is a non-zero element of $M$ that takes $x_{1}$ into zero, a contradiction to $x_{1} \beta \neq 0$ and Lemma 3 .

In the proof of Lemma 2 the case $|G|<4$ was completely investigated so we may take $|G| \geqq 4$.

Suppose $\left|G_{2}\right|>1$. Then there exists distinct elements $x, t \in G_{2}$. Choose a non-zero mapping $f_{c} \in M$ and let $\operatorname{rng} f_{c}=\{0, c\}$. Choose $\gamma_{1}, \gamma_{2} \in T_{0}(G)$ such that $x \gamma_{1}=-c, t \gamma_{1}=b \neq c$, where $b$ is not 0 or $-c-c,(-c) \gamma_{2}=-c, b \gamma_{2}=0$. Note that $b+c$ is not $b, c$, or $-c$ so we can define $(b+c) \gamma_{2}=0$. Then 


$$
(x)\left[\left(\gamma_{1}+f_{c}\right) \gamma_{2}-\gamma_{1} \gamma_{2}\right]=c \text { and }(t)\left[\left(\gamma_{1}+f_{c}\right) \gamma_{2}-\gamma_{1} \gamma_{2}\right]=0 .
$$

But $\left(\gamma_{1}+f_{c}\right) \gamma_{2}-\gamma_{1} \gamma_{2} \in M$ so $t \in G_{1}$, a contradiction.

Thus every element in a minimal right ideal is of the form $\alpha(x, y)$ for some fixed non-zero $x$. We thus have

THEOREM 4. The only minimal right ideals of $T_{0}(G)$ are the $P_{x}$.

A submodule $N$ of the $R$-module $M_{R}$ is called a simple submodule of $M_{R}$ if it contains no proper (nontrivial) submodules. $P$ is a $T_{0}(G)$-module and because of Theorem $1, P_{x}$ is a simple submodule of $P$. The following lemma enables us to characterize all right ideals of $T_{0}(G)$ which are contained in $P$.

Lemma 5. (Beidleman [1, p. 60]). If $M_{R}$ is a near-ring module, then the following are equivalent:

(1) Every submodule is a sum of simple submodules of $M_{R}$,

(2) $M_{R}$ is a sum of simple submodules,

(3) $M_{R}$ is a direct sum of simple submodules,

(4) Every submodule of $M_{R}$ is a direct summand.

THEOREM 5. Every right ideal of $T_{0}(G)$ which is also contained in $P$ is of the form

$$
\sum_{x} \oplus P_{x} \text {, where } x \in H \subseteq G-\{0\} .
$$

The proof follows from Theorems 1 and 4, Lemma 5, and

$$
P=\sum \oplus P_{x}, x \in G-\{0\} .
$$

The above are 'internal' facts about $P$. The following are "external" facts.

$P$ is contained in a maximal right ideal. In fact a standard Zorn's Lemma argument shows that every right ideal is contained in a maximal right ideal. What maximal right ideals are available? For any non-zero $x \in G$ let $A(x)=A(\{x\})$.

Theorem 6. Every $A(x), x \neq 0$, is a maximal right ideal and a maximal $T_{0}(G)$-subgroup.

Proof. Since every right ideal of $T_{0}(G)$ is also a $T_{0}(G)$-subgroup, it suffices to show that $A(x)$ is a maximal $T_{0}(G)$-subgroup of $T_{0}(G)$. Let $B$ be a $T_{0}(G)$-subgroup of $T_{0}(G)$ which properly contains $A(x)$. Choose $\beta \in B$ such that $(x) \beta=$ $b \neq 0$. Take $\eta \in T_{0}(G)$ such that $(b) \eta=x$ and $(t) \eta=0$ for each $t \neq b$. Then $\beta \eta \in B$ and $(x) \beta \eta=x$. Hence, we can assume that $x \beta=x$. Choose $\Phi \in A(x)$ such that $(t) \Phi=-(t) \beta+t$, if $t \neq x$. Then $(t)[\beta+\Phi]=t$ if $t \neq x$ and $(x)[\beta+\Phi]=x$, hence $\beta+\Phi$ is the identity mapping on $G$. Since $\beta+\Phi-\beta \in A(x)$, it follows that $\beta+\Phi \in B$. This shows that $B=T_{0}(G)$ and the theorem follows.

Beidleman [2] defines the radical $J(M)$ of a near-ring module $M_{N}$ as the intersection of all submodules which are maximal as $N$-subgroups. As a consequence 
of Theorem $6, J\left(T_{0}(G)\right)=0$. Note that every right ideal of $T_{0}(G)$ is a $T_{0}(G)$ submodule and conversely.

It is easy to see that $P$ is not contained in any $A(x)$. In fact $P_{x} \cap A(x)=(0)$. By Theorem 6 it follows that $T_{0}(G)=P_{x} \oplus A(x)$. Note that by Theorem 5 $A(x) \cap P=\Sigma \oplus P_{t}, t \neq 0, x$ and hence (by the 2 nd isomorphism theorem for near-ring modules)

$$
T_{0}(G) / P=P+A(x) / P \cong A(x) / P \cap A(x) .
$$

There is an ascending sequence of right ideals containing $P$, however. Let

$$
T_{\lambda}=\left\{f \in T_{0}(G): \mid \text { support } f \mid<\boldsymbol{N}_{\lambda}\right\},
$$

for each ordinal $\lambda$. Here support $f=\{x \in G: x f \neq 0\}$. Each $T_{\lambda}$ is a right ideal and since $P$ is exactly those functions of finite support we have $P=T_{0}$. Also for $\aleph_{\tau}=|G|$

$$
P=T_{0} \subset T_{1} \subset \cdots \subset T_{\tau} \subset T_{0}(G),
$$

where $T_{\tau}$ is contained in a maximal right ideal; the question arises: is $T_{\tau}$ maximal?

The right ideals $A_{n}=A\left(\left\{x_{1}, \cdots, x_{n}\right\}\right)$, where the $x_{n}$ are distinct non-zero elements of $G$, gives rise to an infinite descending chain of right ideals in the case where $G$ is infinite. So $T_{0}(G)$ is not right Artinian for $G$ infinite. The question of whether $T_{0}(G)$ is right Noetherian is open, even for $G$ countable.

The following general lemma will prove useful in the next theorem.

Lemma 6. (Beidleman $\left[1\right.$, p. 54]). If the $N$-module $M_{N}$ is the direct sum of submodules $M_{\lambda}, \lambda \in \Lambda$, then for each $m=m_{\lambda_{1}}+\cdots+m_{\lambda_{n}}$ in $M$, where $m_{\lambda_{i}} \in M_{\lambda_{i}}$, and for each $r \in N$,

$$
m r=m_{\lambda_{1}} r+\cdots+m_{\lambda_{n}} r .
$$

THEOREM 7. If there exists a right ideal $V$ of $T_{0}(G)$ such that $P \oplus V=T_{0}(G)$, then $G$ is finite and $V=(0)$, i.e. $P$ cannot be a proper direct summand.

Proof. If $G$ is finite, then $P=T_{0}(G)$ because of Theorems 3 and 4. Hence, assume $G$ is infinite and $V \neq(0)$. Then

$$
1=\alpha\left(x_{1}, y_{1}\right)+\cdots+\alpha\left(x_{n}, y_{n}\right)+\rho, \rho \in V .
$$

This yields $-y_{i}+x_{i}=x_{i} \rho$ for $i=1,2, \cdots, n$ and for $t \neq x_{i}, t=t \rho$. If for some $j, y_{j} \neq x_{j}$, then we arrive at a contradiction as follows. Choose $\beta_{x} \in T_{0}(G)$ such that $t \beta_{x}=x$, if $t \neq 0$, where $x \notin\left\{x_{1}, \cdots, x_{n}, 0\right\}$. Then using Lemma 6 , and property $D$ we obtain

$$
\beta_{x}=1 \beta_{x}=\sum_{i=1}^{n} \alpha\left(x_{i}, x\right)+\rho \beta_{x}
$$

and $x=x_{j} \beta_{x}=x+\left(-y_{j}+x_{j}\right) \beta_{x}=x+x$, or $x=0$.

Hence

$$
1=\alpha\left(x_{1}, x_{1}\right)+\cdots+\alpha\left(x_{n}, x_{n}\right)+\rho ;
$$


so again using Lemma 6 we have $\alpha(x, x)=\rho \alpha(x, x)$ for each $x \neq x_{i}, i=1, \cdots, n$. But $\rho \alpha(x, x) \in V$, so $0 \neq \rho \alpha(x, x) \in P \cap V$, contrary to $P \cap V=(0)$.

\section{Left ideals}

Let $C^{+}$be the center of $\left(T_{0}(G),+\right)$ and let $C(G)$ be the center of $G$.

Lemma 7. An element $f$ is in $C^{+}$if and only if the image of $f$, written $\operatorname{rng} f$, is in $C(G)$.

Proof. Let $f \in C^{+}$. Consider $(x) f$, for any non-zero $x \in G$. For each $y \in G$ there exists $h \in T_{0}(G)$ such that $(x) h=y$. So

$$
(x) f+y=(x)[f+h]=(x)[h+f]=y+(x) f
$$

and hence $(x) f \in C(G)$.

The converse follows immediately.

Lemma 8. $C^{+}$is a left ideal of $T_{0}(G)$.

Proof. Of course $C^{+}$is a normal subgroup of $\left(T_{0}(G),+\right)$. Since for each $g \in T_{0}(G), f \in C^{+}$:

$$
\operatorname{rng} g f \subseteq \operatorname{rng} f \subseteq C(G),
$$

it follows by Lemma 7 that $g f \in C^{+}$.

Note. For any element $c \in C(G)$ the mapping $f_{c} \in T_{0}(G)$ defined by $x f_{c}=c$, for $x \neq 0$, has its image in $C(G)$ and hence must be in $C^{+}$. So if $G$ has a nontrivial center then $C^{+} \neq(0)$; moreover $\left(T_{0}(G),+\right)$ is centerless if and only if $G$ is centerless.

Next left ideals of $T_{0}(G)$ will be classified in terms of normal subgroups of $G$. Let $L$ be a left $T_{0}(G)$-subgroup of $T_{0}(G)$. Define $G L=\{x l \mid x \in G, l \in L\}$.

Lemma 9. (a) If $L$ is a left $T_{0}(G)$-subgroup of $T_{0}(G)$, then $G L$ is a subgroup of $G$.

(b) If $L$ is a left ideal of $T_{0}(G)$, then $G L$ is a normal subgroup of $G$.

Proof. (a) Let $x_{i} l_{i} \in G L, i=1,2$. Then there exists $f \in T_{0}(G)$ such that $x_{2} f=x_{1}$. Thus

$$
\left(x_{1}\right) l_{1}-\left(x_{2}\right) l_{2}=\left(x_{2}\right) f l_{1}-\left(x_{2}\right) l_{2}=\left(x_{2}\right)\left[f l_{1}-l_{2}\right] \text {. }
$$

Since $f l_{1} \in L$ it follows that $f l_{1}-l_{2} \in L$, hence $\left(x_{1}\right) l_{1}-\left(x_{2}\right) l_{2} \in G L$. This shows $G L$ is a subgroup of $G$.

(b) Let $(x) l \in G L$ and $g \in G$. Consider $g+(x) l-g$. There exists $h \in T_{0}(G)$ such that $(x) h=g$, hence

$$
g+(x) l-g=(x) h+(x) l-(x) h=(x)[h+l-h] .
$$


Now $h+l-h \in L$, hence $G L$ is a normal subgroup of $G$.

To each left ideal $L$ there corresponds a normal subgroup $G L$. This correspondence need not be one-to-one. Note that $G L=(0)$ if and only if $L=(0)$ and $G T_{0}(G)=G$. It is possible for $G L=G$ and yet $L$ be a proper left ideal, as the following example illustrates.

EXAMPLE. Let $G$ be infinite and define

$$
B_{\lambda}=\left\{f \in T_{0}(G):|\operatorname{rng} f|<\boldsymbol{\aleph}_{\lambda}\right\} .
$$

For each ordinal $\lambda, B_{\lambda}$ is a left $T_{0}(G)$-subgroup. Consider $G$ abelian and $\boldsymbol{\aleph}_{\lambda} \leqq|G|$; then $B_{\lambda}$ is a proper left ideal of $T_{0}(G)$ and satisfies $G B_{\lambda}=G$. The latter is clear since for each non-zero $a \in G$ the function $x f_{a}=0$ if $x \neq a, a f_{a}=a$ is in $B_{\lambda}$. For $\boldsymbol{\aleph}_{\alpha}=|G|$ we have the proper ascending chain of left ideals.

$$
(0) \subset B_{0} \subset B_{1} \subset \cdots \subset B_{\alpha} \subset T_{0}(G) \text {. }
$$

For any group $G$ if $z \in G L$, then for each non-zero $x \in G, \alpha(x, z) \in L$. Some consequences and their immediate corollaries are given in the following

THEOREM 8. Let $L$ be a left $T_{0}(G)$-subgroup.

(1) If $G L=G$, then $P \subseteq L$,

(2) If $G L=G$ and $G$ is finite, then $L=T_{0}(G)$,

(3) If $G$ is a finite simple group, then $T_{0}(G)$ has no proper left ideals,

(4) If $L$ is also a right $T_{0}(G)$-subgroup, then $P \subseteq L$ and if in addition $G$ is finite, then $L=T_{0}(G)$,

(5) If $G$ is finite, then $T_{0}(G)$ is a simple near-ring,

(6) If $G$ is a simple group, then $P$ is contained in every non-zero left ideal of $T_{0}(G)$.

To each normal subgroup $N$ of $G$ there corresponds a left ideal of $T_{0}(G)$. Consider

$$
L_{N}=\left\{l \in T_{0}(G): G l \subseteq N\right\} .
$$

Lemma 10. $L_{N}$ is a left ideal of $T_{0}(G)$.

LEMMA 11. If $M$ and $N$ are normal subgroups of $G$ and $M \subseteq N$, then $L_{M} \subseteq L_{N}$. If $L_{1}$ and $L_{2}$ are left ideals of $T_{0}(G)$ and $L_{1} \subseteq L_{2}$, then $G L_{1} \subseteq G L_{2}$.

The proof of each of these lemmas follows directly from the definitions.

The inter-relationship between the normal subgroups given by Lemma 9 and the left ideals given by Lemma 10 is investigated next.

Starting with a left ideal $L$ we pass to a normal subgroup $G L$ and then a left ideal $L_{G L}$. We have $L \subseteq L_{G L}$. Again using Lemma 9 we obtain the normal subgroups $G L$ and $G L_{G L}$ where $G L \subseteq G L_{G L}$ by Lemma 11. But $G L_{G L} \subseteq G L$ by definition so $G L=G L_{G L}$. 
Next start with any normal subgroup $N$ of $G$, then pass to the left ideal $L_{N}$ and the normal subgroup $G L_{N}$. As in the previous paragraph, but using $L_{N}$ as the left ideal, this yields $L_{N} \subseteq L_{M}$, where $M=G L_{N}$. But $M=G L_{N} \subseteq N$, so $L_{M} \subseteq L_{N}$ and hence $L_{N}=L_{M}$.

\section{References}

[1] J. C. Beidleman, On near-rings and near-ring modules (Doctoral Dissertation, Pennsylvania State University, 1964).

[2] J. C. Beidleman, 'A radical for near-ring modules', Michigan Math. J., 12 (1965), 377-383.

[3] G. Berman and R. J. Silverman, 'Simplicity of near-rings of transformations', Proc. Amer. Math. Soc., 10 (1959), 456-459.

[4] D. W. Blackett, Simple and semi-simple near-rings (Doctoral Dissertation, Princeton University, 1950).

[5] J. J. Malone Jr. and H. E. Heatherly, 'Some near-ring embeddings', Quart. J. Math. Oxford (2), 20 (1969), $81-85$.

University of Southwestern Louisiana

Lafayette, Louisiana 